\title{
Specific water structure in a geo-inspired nanotube and interrelated dynamics
}

\author{
Geoffrey Monet ${ }^{1}$, Arianna D'Angelo ${ }^{1,2}$, Erwan Paineau ${ }^{1}$, Gilberto teobaldi ${ }^{3}$, Stéphane Rols ${ }^{2}$, Pascale Launois ${ }^{1}$ \\ ${ }^{1}$ Laboratoire de Physique des Solides, CNRS, université Paris-Saclay, Orsay, France; \\ ${ }^{2}$ Institut Laue-Langevin, Grenoble, France; \\ ${ }^{3}$ STFC, Daresbury laboratory, UK; \\ pascale.launois@universite-paris-saclay.fr
}

Water in confined geometries or near surfaces exhibits radically different properties depending on its environment. The physics of the new states of water is often discussed in terms of the distortion of its tetrahedral local symmetry and/or in terms of the frustration of its hydrogen-bond network. At the extreme opposite of these views is the investigation of the physical properties of the molecules when they are isolated from each other. This is experimentally challenging and related studies are scarce $[1,2,3]$.

By combined use of wide-angle X-ray scattering, inelastic neutron scattering, density functional theory (DFT) and DFT molecular dynamics (MD) simulations, we investigated the structure, dynamics and stability of the water wetting-layer in single-walled aluminogermanate imogolite nanotubes [4]: an archetypal system for synthetically controllable and monodisperse nanochannels. We demonstrate that the water wetting-layer is strongly bound and solid-like up to $300 \mathrm{~K}$ under atmospheric pressure. Atomic-scale characterisation of the wetting-layer reveals organisation of the $\mathrm{H}_{2} \mathrm{O}$ molecules in a curved triangular sublattice stabilised by the formation of three $\mathrm{H}$-bonds to the nanotube's inner surface, with covalent interactions sufficiently strong to promote energetically favourable decoupling of the $\mathrm{H}_{2} \mathrm{O}$ molecules in the adlayer. The dynamics of the water molecules is markedly different from that of bulk water. It is dictated by its interactions with the nanotube and conversely, this structural water impacts the dynamics of the nanotube. The peculiar dynamics of hydroxyl groups in dry imogolite nanotubes will also be discussed based on MD simulations and on elastic neutron scattering measurements.

The above results point the way to a systematic study of the effects of different water loadings and water-soluble reactants in complex oxide-based nanoreactors starting from, but not limited to, the imogolite family.

[1] C. Beduz et al., PNAS 109, 12894 (2012)

[2] A.I. Kolesnikov et al., Phys. Rev. Lett. 116, 167802 (2016)

[3] T.R. Prisk et al., Phys. Rev. Lett. 120, 196001 (2018)

[4] G. Monet et al., Nanoscale Adv. 2, 1869 (2020)

Keywords: structure, dynamics, DFT, nanoconfinement 